\title{
ON THE DARBOUX INTEGRABILITY OF THE LOGARITHMIC GALACTIC POTENTIALS
}

\author{
JAUME LLIBRE ${ }^{1}$ AND CLÀUDIA VALLS ${ }^{2}$
}

\begin{abstract}
We study the logarithmic Hamiltonians $H=\left(p_{x}^{2}+p_{y}^{2}\right) / 2+\log \left(1+x^{2}+\right.$ $\left.y^{2} / q^{2}\right)^{1 / 2}$, which appears in the study of the galactic dynamics. We characterize all the invariant algebraic hypersurfaces and all exponential factors of the Hamiltonian system with Hamiltonian $H$. We prove that this Hamiltonian system is completely integrable with Darboux first integrals if and only if $q= \pm 1$.
\end{abstract}

\section{Introduction AND STATEMENT OF the MAIN RESUlts}

The potential

$$
V=\frac{1}{2} \log \left(R^{2}+x^{2}+\frac{y^{2}}{q^{2}}\right)
$$

where $q \in \mathbb{R} \backslash\{0\}$ is called the logarithmic potential. It has an absolute minimum and reflection symmetry with respect to both axes. This potential is relevant in problems of galactic dynamics as a model for elliptical galaxies. More precisely, it is a model of a core embedded in a dark matter halo, with $R$ being the core radius. Without loss of generality we can assume that $R=1$, and the energy can take any non-negative value. The parameter $q$ is the ellipticity of the potential, which ranges in the interval $0.6 \leq q \leq 1$. Lower values of $q$ have no physical meaning and greater values of $q$ are equivalent to reverse the role of the coordinate axes. In this paper, to make a complete and deep study of the Darboux integrability of such a potentials we will consider that $q \in \mathbb{R} \backslash\{0\}$. This model has been intensively investigated from different dynamical and physical point of views by several authors, see for instance $[3,7,11,12,13]$.

We consider the logarithmic Hamiltonian

$$
H=\frac{1}{2}\left(p_{x}^{2}+p_{y}^{2}\right)+\frac{1}{2} \log \left(1+x^{2}+\frac{y^{2}}{q^{2}}\right), \quad q \in \mathbb{R} \backslash\{0\},
$$

its Hamiltonian system is

$$
\begin{aligned}
\dot{x} & =-p_{x}, \\
\dot{y} & =-p_{y}, \\
\dot{p}_{x} & =\frac{x}{1+x^{2}+y^{2} / q^{2}}, \\
\dot{p}_{y} & =\frac{y}{q^{2}\left(1+x^{2}+y^{2} / q^{2}\right)},
\end{aligned}
$$

Key words and phrases. Celestial Mechanics, Galaxy evolution. 
where the dot indicates derivative with respect to time $t$. Note that this Hamiltonian system (1) has two degrees of freedom.

The main aim of this paper is to study the existence of first integrals of system (1). The vector field $X$ associated to system (1) is

$$
X=-p_{x} \frac{\partial}{\partial x}-\frac{p_{y}}{q} \frac{\partial}{\partial y}+\frac{x}{1+x^{2}+y^{2} / q^{2}} \frac{\partial}{\partial p_{x}}+\frac{y}{q^{2}\left(1+x^{2}+y^{2} / q^{2}\right)} \frac{\partial}{\partial p_{y}} .
$$

Let $U \subset \mathbb{R}^{2}$ be an open set. We say that the non-constant function $F: \mathbb{R}^{2} \rightarrow \mathbb{R}$ is a first integral of a vector field $X$ on $U$, if $F\left(x(t), y(t), p_{x}(t), p_{y}(t)\right)=$ constant for all values of $t$ for which the solution $\left(x(t), y(t), p_{x}(t), p_{y}(t)\right)$ of $X$ is defined on $U$. Clearly $F$ is a first integral of $X$ on $U$ if and only if $X F=0$ on $U$.

We say that the functions $F_{1}, \ldots, F_{n}$ are in involution if $\left\{F_{i}, F_{j}\right\}=0$ for all $i \neq j$, where $\{\cdot, \cdot\}$ denotes the Poisson bracket. Moreover, they are independent if the oneforms $d F_{1}, \ldots, d F_{n}$ are linearly independent over a full Lebesgue measure subset of the common definition domain of $F_{j}$ for $j=1, \ldots, n$. By definition, a Hamiltonian system with $n$ degrees of freedom having $n$ independent first integrals in involution is completely integrable, see for more details [1].

Note that system (1) is completely integrable, if and only if there exists a first integral linearly independent and in involution with $H$. We have the following result, whose proof follows by direct computations.

Proposition 1. When $q= \pm 1$ the Hamiltonian system (1) is completely integrable with the first integrals $H$ and $H_{1}=y p_{x}-x p_{y}$.

From now on we will restrict to the case $q \neq \pm 1$. Doing the change of time $d t=\left(1+x^{2}+y^{2} / q^{2}\right) d s$, system (1) becomes

$$
\begin{aligned}
x^{\prime} & =-p_{x}\left(1+x^{2}+y^{2} / q^{2}\right), \\
y^{\prime} & =-p_{y}\left(1+x^{2}+y^{2} / q^{2}\right), \\
p_{x}^{\prime} & =x \\
p_{y}^{\prime} & =\frac{y}{q^{2}}
\end{aligned}
$$

where the prime denotes derivative with respect to the new time $s$.

Taking the notation $Y=y / q, Q=1 / q^{2}>0, P_{Y}=q p_{y}$ we get

$$
\begin{aligned}
x^{\prime} & =-p_{x}\left(1+x^{2}+Y^{2}\right), \\
Y^{\prime} & =-Q P_{Y}\left(1+x^{2}+Y^{2}\right), \\
p_{x}^{\prime} & =x, \\
P_{Y}^{\prime} & =Y .
\end{aligned}
$$


We write the previous system again as

$$
\begin{aligned}
x^{\prime} & =-p_{x}\left(1+x^{2}+y^{2}\right), \\
y^{\prime} & =-Q p_{y}\left(1+x^{2}+y^{2}\right), \\
p_{x}^{\prime} & =x, \\
p_{y}^{\prime} & =y .
\end{aligned}
$$

The vector field associated to system (2) is

$$
X=-p_{x}\left(1+x^{2}+y^{2}\right) \frac{\partial}{\partial x}-Q p_{y}\left(1+x^{2}+y^{2}\right) \frac{\partial}{\partial y}+x \frac{\partial}{\partial p_{x}}+y \frac{\partial}{\partial p_{y}} .
$$

Note that system (2) has the first integral

$$
H_{0}=\left(1+x^{2}+y^{2}\right) e^{p_{x}^{2}+Q p_{y}^{2}} .
$$

From now on $Q \neq 1$.

The aim of this paper is to study the existence of additional first integrals of system (2) which are linearly independent with $H_{0}$ and that can be described by functions of Darboux type (see (7)). Note that one of the main tools for studying the dynamics of the differential system (2) is to know the existence of an additional independent first integral for some values of the parameter $Q>0$. In general, for a given differential system it is a difficult problem to determine the existence or nonexistence of first integrals.

First we study the existence of first integrals of system (2) given by polynomials.

A polynomial first integral $f=f\left(x, y, p_{x}, p_{y}\right)$ of system $(2)$ is a polynomial in the variables $x, y, p_{x}$ and $p_{y}$ such that

$$
-p_{x}\left(1+x^{2}+y^{2}\right) \frac{\partial f}{\partial x}-Q p_{y}\left(1+x^{2}+y^{2}\right) \frac{\partial f}{\partial y}+x \frac{\partial f}{\partial p_{x}}+y \frac{\partial f}{\partial p_{y}}=0 .
$$

The first main result is the following.

Theorem 2. System (2) with $Q \neq 1$ has no polynomial first integrals.

The proof of Theorem 2 is given in section 2 .

A rational first integral of system (2) is a rational function $f$ satisfying (4).

Theorem 3. System (2) with $Q \neq 1$, has no rational first integrals.

The proof of Theorem 3 is given in section 4 .

To prove Theorem 3 we will use the Darboux theory of integrability. The Darboux theory of integrability in dimension 4 is based on the existence of invariant algebraic hypersurfaces (or Darboux polynomials). For more details see $[4,5,6]$. This theory is one of the best theories for studying the existence of first integrals for the polynomial differential systems.

A Darboux polynomial of system (2) is a polynomial $f \in \mathbb{C}\left[x, y, p_{x}, p_{y}\right] \backslash \mathbb{C}$ such that

$$
-p_{x}\left(1+x^{2}+y^{2}\right) \frac{\partial f}{\partial x}-Q p_{y}\left(1+x^{2}+y^{2}\right) \frac{\partial f}{\partial y}+x \frac{\partial f}{\partial p_{x}}+y \frac{\partial f}{\partial p_{y}}=K f,
$$


for some polynomial

$$
\begin{aligned}
K= & \alpha_{0}+\alpha_{1} x+\alpha_{2} y+\alpha_{3} p_{x}+\alpha_{y} p_{y}+\alpha_{5} x^{2}+\alpha_{6} x y+\alpha_{7} x p_{x}+\alpha_{8} x p_{y}+\alpha_{9} y^{2} \\
& +\alpha_{10} y p_{x}+\alpha_{11} y p_{y}+\alpha_{12} p_{x}^{2}+\alpha_{13} p_{x} p_{y}+\alpha_{14} p_{y}^{2},
\end{aligned}
$$

called the cofactor of $f$. Note that $f=0$ is an invariant algebraic hypersurface for the flow of system (2), and a polynomial first integral is a Darboux polynomial with zero cofactor. We note that if $f \notin \mathbb{R}\left[x, y, p_{x}, p_{y}\right] \backslash \mathbb{R}$ is a Darboux polynomial then there exists another Darboux polynomial $\bar{f}$ (the conjugate of $f$ ) with cofactor $\bar{K}$ (the conjugate of $K)$.

Theorem 4. The unique irreducible Darboux polynomial with non-zero cofactor of system (2) with $Q \neq 1$ is $1+x^{2}+y^{2}$.

The proof of Theorem 4 is given in section 3 .

An exponential factor $F=F\left(x, y, p_{x}, p_{y}\right)$ of system (2) is a function of the form $F=\exp \left(g_{0} / g_{1}\right) \notin \mathbb{C}$ with $g_{0}, g_{1} \in \mathbb{C}\left[x, y, p_{x}, p_{y}\right]$ coprime satisfying that

$$
-p_{x}\left(1+x^{2}+y^{2}\right) \frac{\partial F}{\partial x}-Q p_{y}\left(1+x^{2}+y^{2}\right) \frac{\partial F}{\partial y}+x \frac{\partial F}{\partial p_{x}}+y \frac{\partial F}{\partial p_{y}}=L F,
$$

for some polynomial $L=L\left(x, y, p_{x}, p_{y}\right)$ of degree at most 2 , called the cofactor of $F$. We note that if $F \notin \mathbb{R}\left[x, y, p_{x}, p_{y}\right] \backslash \mathbb{R}$ is an exponential factor then there exists another exponential factor $\bar{F}$ (the conjugate of $F$ ) with cofactor $\bar{L}$ (the conjugate of $L)$.

Theorem 5. The unique exponential factors of system (2) with $Q \neq 1$ are $e^{p_{x}}, e^{p_{y}}$, $e^{p_{x}^{2}}, e^{p_{x} p_{y}}, e^{p_{y}^{2}}, e^{y p_{x}-Q x p_{y}}$, and exponential of linear combinations of all the exponents in the previous exponential factors.

The proof of Theorem 5 is given in Section 5 .

A Darboux first integral $G$ of system (2) is a first integral of the form

$$
G_{=} f_{1}^{\lambda_{1}} \cdots f_{p}^{\lambda_{p}} F_{1}^{\mu_{1}} \cdots F_{q}^{\mu_{q}},
$$

where $f_{1}, \ldots, f_{p}$ are Darboux polynomials and $F_{1}, \ldots, F_{q}$ are exponential factors and $\lambda_{j}, \mu_{k} \in \mathbb{C}$ for $j=1, \ldots, p$ and $k=1, \ldots, q$. Note that the Darboux first integral always is a real function due to the fact that if there are complex polynomials or complex exponential factors, then always also appear their conjugates.

Theorem 6. The unique Darboux first integrals of system (2) with $Q \neq 1$ are functions of Darboux type of $H_{0}$.

The proof of Theorem 6 is given in section 6 .

In short, from Proposition 1 and Theorem 6 we have the following result.

Corollary 7. The Hamiltonian system (1) is completely integrable with Darboux first integrals if and only if $q= \pm 1$. 


\section{Polynomial first integrals: Proof of Theorem 2}

Let $f$ be a polynomial first integral of system (2). Without loss of generality we can assume that it has no constant term. Then $f$ satisfies (4). We write $f$ as $f=\sum_{j=0}^{n} f_{j}\left(x, y, p_{x}, p_{y}\right)$ where each $f_{j}$ is a homogeneous polynomial of degree $j$ in each variables $x, y, p_{x}$ and $p_{y}$. We can assume that $f_{n} \neq 0$ with $n>0$. We have that the terms of degree $n+2$ in (4) satisfy

$$
\left(x^{2}+y^{2}\right)\left(p_{x} \frac{\partial f_{n}}{\partial x}+Q p_{y} \frac{\partial f_{n}}{\partial y}\right)=0
$$

Solving it we get

$$
f_{n}=K_{n}\left(p_{x}, p_{y}, y p_{x}-Q x p_{y}\right),
$$

where $K_{n}$ is any function in the variables $p_{x}, p_{y}$ and $y p_{x}-Q x p_{y}$. Since $f_{n}$ must be a homogeneous polynomial of degree $n$ we must have

$$
f_{n}=\sum_{j_{1}+j_{2}+2 m=n} a_{j_{1}, j_{2}, m} p_{x}^{j_{1}} p_{y}^{j_{2}}\left(y p_{x}-Q x p_{y}\right)^{m}, \quad a_{j_{1}, j_{2}, m} \in \mathbb{R} .
$$

Now the terms of degree $n$ in (4) satisfy

$$
\begin{aligned}
& \left(x^{2}+y^{2}\right)\left(p_{x} \frac{\partial f_{n-2}}{\partial x}+Q p_{y} \frac{\partial f_{n-2}}{\partial y}\right)=-p_{x} \frac{\partial f_{n}}{\partial x}-Q p_{y} \frac{\partial f_{n}}{\partial y}+x \frac{\partial f_{n}}{\partial p_{x}}+y \frac{\partial f_{n}}{\partial p_{y}} \\
& =x \sum_{j_{1}+j_{2}+2 m=n} j_{1} a_{j_{1}, j_{2}, m} p_{x}^{j_{1}-1} p_{y}^{j_{2}}\left(y p_{x}-Q x p_{y}\right)^{m} \\
& \quad+y \sum_{j_{1}+j_{2}+2 m=n} j_{2} a_{j_{1}, j_{2}, m} p_{x}^{j_{1}} p_{y}^{j_{2}-1}\left(y p_{x}-Q x p_{y}\right)^{m} \\
& \quad+(1-Q) x y \sum_{j_{1}+j_{2}+2 m=n} m a_{j_{1}, j_{2}, m} p_{x}^{j_{1}} p_{y}^{j_{2}}\left(y p_{x}-Q x p_{y}\right)^{m-1} .
\end{aligned}
$$

Now we introduce the variable

$$
Y=y p_{x}-Q x p_{y} \quad \text { and } \quad y=\frac{Y+Q x p_{y}}{p_{x}} .
$$

Then we can rewrite the right-hand side of (10) in the variables $\left(x, Y, p_{x}, p_{y}\right)$ as

$$
\begin{aligned}
x & \sum_{j_{1}+j_{2}+2 m=n}\left(j_{1}+Q j_{2}+(1-Q) m\right) a_{j_{1}, j_{2}, m} p_{x}^{j_{1}-1} p_{y}^{j_{2}} Y^{m} \\
& +\sum_{j_{1}+j_{2}+2 m=n} j_{2} a_{j_{1}, j_{2}, m} p_{x}^{j_{1}-1} p_{y}^{j_{2}-1} Y^{m+1} \\
& +(1-Q) Q x^{2} \sum_{j_{1}+j_{2}+2 m=n} m a_{j_{1}, j_{2}, m} p_{x}^{j_{1}-1} p_{y}^{j_{2}+1} Y^{m-1} .
\end{aligned}
$$


Note that in these new variables, if we set $\tilde{f}_{n-2}\left(x, Y, p_{x}, p_{y}\right)=f_{n-2}\left(x, y, p_{x}, p_{y}\right)$ then we can rewrite (10) as

$$
\begin{aligned}
\frac{\partial \tilde{f}_{n-2}}{\partial x}= & \frac{x}{x^{2} p_{x}^{2}+\left(Y+Q x p_{y}\right)^{2}} \sum_{j_{1}+j_{2}+2 m=n}\left(j_{1}+Q j_{2}+(1-Q) m\right) a_{j_{1}, j_{2}, m} p_{x}^{j_{1}} p_{y}^{j_{2}} Y^{m} \\
& +\frac{1}{x^{2} p_{x}^{2}+\left(Y+Q x p_{y}\right)^{2}} \sum_{j_{1}+j_{2}+2 m=n} j_{2} a_{j_{1}, j_{2}, m} p_{x}^{j_{1}} p_{y}^{j_{2}-1} Y^{m+1} \\
& \frac{(1-Q) Q x^{2}}{x^{2} p_{x}^{2}+\left(Y+Q x p_{y}\right)^{2}} \sum_{j_{1}+j_{2}+2 m=n} m a_{j_{1}, j_{2}, m} p_{x}^{j_{1}} p_{y}^{j_{2}+1} Y^{m-1} .
\end{aligned}
$$

Using the integrals

$$
\begin{aligned}
\int \frac{d x}{x^{2} p_{x}^{2}+\left(Y+Q x p_{y}\right)^{2}}= & \frac{1}{p_{x} Y} \arctan \left(\frac{x p_{x}^{2}+p_{y}^{2} Q^{2} x+p_{y} Q Y}{p_{x} Y}\right), \\
\int \frac{x}{x^{2} p_{x}^{2}+\left(Y+Q x p_{y}\right)^{2}} d x= & \frac{1}{2\left(p_{x}^{3}+p_{y}^{2} Q^{2} p_{x}\right)}\left(p_{x} \log \left(p_{x}^{2} x^{2}+\left(p_{y} Q x+Y\right)^{2}\right)\right. \\
& \left.-2 p_{y} Q \arctan \left(\frac{x p_{x}^{2}+p_{y} Q\left(p_{y} Q x+Y\right)}{p_{x} Y}\right)\right), \\
\int \frac{x^{2}}{x^{2} p_{x}^{2}+\left(Y+Q x p_{y}\right)^{2}} d x= & \frac{1}{p_{x}\left(p_{x}^{2}+p_{y}^{2} Q^{2}\right)^{2}}\left(\left(p_{y}^{2} Q^{2} Y-p_{x}^{2} Y\right)\right. \\
& \arctan \left(\frac{x p_{x}^{2}+p_{y} Q\left(p_{y} Q x+Y\right)}{p_{x} Y}\right)+p_{x}\left(\left(p_{x}^{2}+p_{y}^{2} Q^{2}\right) x\right. \\
& \left.\left.-p_{y} Q Y \log \left(p_{x}^{2} x^{2}+\left(p_{y} Q x+Y\right)^{2}\right)\right)\right),
\end{aligned}
$$

we get

$$
\begin{aligned}
\tilde{f}_{n-2}= & \frac{1}{2\left(p_{x}^{2}+p_{y}^{2} Q^{2}\right)^{2}} \sum_{j_{1}+j_{2}+2 m=n} a_{j_{1}, j_{2}, m} p_{x}^{j_{1}-1} p_{y}^{j_{2}-1} Y^{m-1}\left(2 \left(\left(j_{2}+2 m\right) p_{y}^{2} Q^{2} p_{x}^{2}\right.\right. \\
& \left.+j_{2} p_{x}^{4}-\left(j_{1}+2 m\right) p_{y}^{2} Q p_{x}^{2}-j_{1} p_{y}^{4} Q^{3}\right) Y \arctan \left(\frac{x p_{x}^{2}+p_{y}^{2} Q^{2} x+p_{y} Q Y}{p_{x} Y}\right) \\
& +p_{x} p_{y}\left(\left(j_{2}+m\right) p_{y}^{2} Q^{3}+\left(j_{1}-m\right) p_{y}^{2} Q^{2}+\left(j_{2}-m\right) p_{x}^{2} Q+\left(j_{1}+m\right) p_{x}^{2}\right) Y \\
& \left.\log \left(p_{x}^{2} x^{2}+\left(p_{y} Q x+Y\right)^{2}\right)-2 m p_{y}(Q-1) Q\left(p_{x}^{2}+p_{y}^{2} Q^{2}\right) x\right)+K_{n-2}\left(p_{x}, p_{y}, Y\right) .
\end{aligned}
$$

Since $f_{n-2}$ must be a polynomial, in particular, we must have that the part with arctan must be zero. Then,

$$
\sum_{j_{1}+j_{2}+2 m=n} a_{j_{1}, j_{2}, m} p_{x}^{j_{1}} p_{y}^{j_{2}} Y^{m}\left(\left(j_{2}+2 m\right) p_{y}^{2} Q^{2} p_{x}^{2}+j_{2} p_{x}^{4}-\left(j_{1}+2 m\right) p_{y}^{2} Q p_{x}^{2}-j_{1} p_{y}^{4} Q^{3}\right)=0 .
$$

This implies that either $a_{j_{1}, j_{2}, m}=0$, or

$$
j_{1}=0, \quad j_{2}=0, \quad\left(j_{2}+2 m\right) Q^{2}-\left(j_{1}+2 m\right) Q=0 .
$$


Hence, since $Q(Q-1) \neq 0$ we get that either $a_{j_{1}, j_{2}, m}=0$ or $j_{1}=j_{2}=m=0$. In the first case $f_{n}=0$, and in the second one $n=0$. So, in both cases we have a contradiction with the fact that $f$ is a polynomial first integral.

\section{Darboux polynomials with non-Zero cofactor: Proof of Theorem 4}

We consider a Darboux polynomial with non-zero cofactor. We write it as $f=$ $\sum_{j=0}^{n} f_{j}\left(x, y, p_{x}, p_{y}\right)$ where each $f_{j}$ is a homogeneous polynomial of degree $j$ in each variables $x, y, p_{x}$ and $p_{y}$. Without loss of generality we can assume that $f_{n} \neq 0$ with $n>0$. We have that the terms of degree $n+2$ in (5) satisfy

$$
\begin{aligned}
& -\left(x^{2}+y^{2}\right)\left(p_{x} \frac{\partial f_{n}}{\partial x}+Q p_{y} \frac{\partial f_{n}}{\partial y}\right)=\left(\alpha_{5} x^{2}+\alpha_{6} x y+\alpha_{7} x p_{x}+\alpha_{8} x p_{y}+\alpha_{9} y^{2}\right. \\
& \left.+\alpha_{10} y p_{x}+\alpha_{11} y p_{y}+\alpha_{12} p_{x}^{2}+\alpha_{13} p_{x} p_{y}+\alpha_{14} p_{y}^{2}\right) f_{n} .
\end{aligned}
$$

Solving the differential equation in (13) we have

$$
\begin{gathered}
f_{n}=K_{n}\left(p_{x}, p_{y}, y p_{x}-Q x p_{y}\right) \exp \left(\frac{-\left(\alpha_{5} p_{x}^{2}+Q p_{y}\left(\alpha_{6} p_{x}+\alpha_{9} Q p_{y}\right)\right) x}{p_{x}\left(p_{x}^{2}+Q^{2} p_{y}^{2}\right)}\right) \\
\quad \exp \left(\frac{-T_{1} \arctan \left(\frac{p_{x} x+Q y p_{y}}{Q x p_{y}-y p_{x}}\right)}{\left(Q x p_{y}-y p_{x}\right)\left(p_{x}^{2}+Q^{2} p_{y}^{2}\right)^{2}}\right)\left(p_{x}^{2}\left(x^{2}+y^{2}\right)\right)^{\frac{-T_{2}}{2\left(p_{x}^{2}+Q^{2} p_{y}^{2}\right)^{2}}},
\end{gathered}
$$

where

$$
\begin{aligned}
T_{1}= & \left(\alpha_{14} p_{y}^{2}+\alpha_{13} p_{x} p_{y}+\alpha_{12} p_{x}^{2}\right)\left(p_{x}^{2}+Q^{2} p_{y}^{2}\right)^{2} \\
& +\left(Q x p_{y}-y p_{x}\right)\left(p_{x}^{2}+Q^{2} p_{y}^{2}\right)\left(-\alpha_{10} p_{x}^{2}-\left(\alpha_{11}-Q \alpha_{7}\right) p_{x} p_{y}+\alpha_{8} Q p_{y}^{2}\right) \\
& +\left(\left(\alpha_{5}-\alpha_{9}\right) p_{x}^{2}+2 \alpha_{6} Q p_{x} p_{y}+\left(\alpha_{9}-\alpha_{5}\right) Q^{2} p_{y}^{2}\right)\left(y p_{x}-Q x p_{y}\right), \\
T_{2}= & \left(\alpha_{7} p_{x}^{2}+\left(\alpha_{8}+\alpha_{10} Q\right) p_{x} p_{y}+\alpha_{11} Q p_{y}^{2}\right)\left(p_{x}^{2}+Q^{2} p_{y}^{2}\right) \\
& +\left(2 Q\left(\alpha_{9}-\alpha_{5}\right) p_{x} p_{y}+\alpha_{6}\left(p_{x}^{2}-Q^{2} p_{y}^{2}\right)\right)\left(y p_{x}-Q x p_{y}\right) .
\end{aligned}
$$

Since $f_{n}$ must be a polynomial, introducing the change of variables in (11), the part of the first exponential must be zero. Then $\alpha_{5}=\alpha_{6}=\alpha_{9}=0$. Then, $T_{1}$ reduces to

$$
\begin{aligned}
& \left(\alpha_{14} p_{y}^{2}+\alpha_{13} p_{x} p_{y}+\alpha_{12} p_{x}^{2}\right)\left(p_{x}^{2}+Q^{2} p_{y}^{2}\right)^{2}+\left(Q x p_{y}-y p_{x}\right)\left(p_{x}^{2}+Q^{2} p_{y}^{2}\right) \\
& \left(-\alpha_{10} p_{x}^{2}-\left(\alpha_{11}-Q \alpha_{7}\right) p_{x} p_{y}+\alpha_{8} Q p_{y}^{2}\right)= \\
& \alpha_{12} p_{x}^{6}+\alpha_{13} p_{y} p_{x}^{5}+\alpha_{10} y p_{x}^{5}+\alpha_{14} p_{y}^{2} p_{x}^{4}+2 \alpha_{12} p_{y}^{2} Q^{2} p_{x}^{4}-\alpha_{10} p_{y} Q x p_{x}^{4} \\
& +p_{y}\left(\alpha_{11}-\alpha_{7} Q\right) y p_{x}^{4}+2 \alpha_{13} p_{y}^{3} Q^{2} p_{x}^{3}-p_{y}^{2} Q\left(\alpha_{11}-\alpha_{7} Q\right) x p_{x}^{3} \\
& -p_{y}^{2} Q\left(\alpha_{8}-\alpha_{10} Q\right) y p_{x}^{3}+p_{y}^{4} Q^{2}\left(\alpha_{12} Q^{2}+2 \alpha_{14}\right) p_{x}^{2}+p_{y}^{3} Q^{2}\left(\alpha_{8}-\alpha_{10} Q\right) x p_{x}^{2} \\
& +p_{y}^{3} Q^{2}\left(\alpha_{11}-\alpha_{7} Q\right) y p_{x}^{2}+\alpha_{13} p_{y}^{5} Q^{4} p_{x}-p_{y}^{4} Q^{3}\left(\alpha_{11}-\alpha_{7} Q\right) x p_{x} \\
& -\alpha_{8} p_{y}^{4} Q^{3} y p_{x}+\alpha_{14} p_{y}^{6} Q^{4}+\alpha_{8} p_{y}^{5} Q^{4} x
\end{aligned}
$$

Equating the coefficients of the same monomials in the previous equation, we get

$$
\alpha_{8}=\alpha_{10}=\alpha_{12}=\alpha_{13}=\alpha_{14}=0,
$$

and $\alpha_{11}=Q \alpha_{7}$. Then, $T_{2}=\alpha_{7}\left(p_{x}^{2}+Q^{2} p_{y}^{2}\right)^{2}$, which yields $\alpha_{7}=-2 m$ with $m \in \mathbb{N}$. Hence

$$
K=\alpha_{0}+\alpha_{1} x+\alpha_{2} y+\alpha_{3} p_{x}+\alpha_{4} p_{y}-2 m\left(x p_{x}+Q y p_{y}\right),
$$


and

$$
f_{n}=K\left(p_{x}, p_{y}, y p_{x}-Q x p_{y}\right)\left(x^{2}+y^{2}\right)^{m} .
$$

Since $f_{n}$ must have degree $n$ we get

$$
f_{n}=\left(x^{2}+y^{2}\right)^{m} \sum_{j_{1}+j_{2}+2 j_{3}=n-2 m} a_{j_{1}, j_{2}, j_{3}} p_{x}^{j_{1}} p_{y}^{j_{2}}\left(y p_{x}-Q x p_{y}\right)^{j_{3}}, \quad a_{j_{1}, j_{2}, j_{3}} \in \mathbb{C} .
$$

Computing the terms of degree $n+1$ in (5) we get

$$
\begin{aligned}
& -\left(x^{2}+y^{2}\right)\left(p_{x} \frac{\partial f_{n-1}}{\partial x}+Q p_{y} \frac{\partial f_{n-1}}{\partial y}\right)=-2 m\left(x p_{x}+Q y p_{y}\right) f_{n-1} \\
& +\left(\alpha_{1} x+\alpha_{2} y+\alpha_{3} p_{x}+\alpha_{4} p_{y}\right) f_{n} .
\end{aligned}
$$

Now, proceeding as in the proof of Theorem 2, introducing the change of variables (11), solving it and then going back to the old variables we obtain

$$
\begin{aligned}
f_{n-1}= & K_{n-1}\left(p_{x}, p_{y}, y p_{x}-Q x p_{y}\right)\left(x^{2}+y^{2}\right)^{m}+\frac{\left(x^{2}+y^{2}\right)^{m}}{2\left(p_{x}^{2}+p_{y}^{2} Q^{2}\right)}\left(2 \left(\alpha_{3}\left(p_{x}^{2}+p_{y}^{2} Q^{2}\right) p_{x}^{2}\right.\right. \\
& \left.+\alpha_{4} p_{y}\left(p_{x}^{2}+p_{y}^{2} Q^{2}\right) p_{x}+\left(\alpha_{2}\left(p_{x}^{2}-p_{y}^{2} Q^{2}(x-1)\right)-\alpha_{1} p_{x} p_{y} Q\right)\left(p_{x} y-p_{y} Q x\right)\right) \\
& \left.\arctan \left(\frac{p_{x} x+p_{y} Q y}{p_{y} Q x-p_{x} y}\right)-p_{x}\left(\alpha_{1} p_{x}+\alpha_{2} p_{y} Q x\right)\left(p_{x} y-p_{y} Q x\right) \log \left(p_{x}^{2}\left(x^{2}+y^{2}\right)\right)\right) \\
& \sum_{j_{1}+j_{2}+2 j_{3}=n-2 m} a_{j_{1}, j_{2}, j_{3}} p_{x}^{j_{1}-1} p_{y}^{j_{2}}\left(p_{x} y-p_{y} Q x\right)^{j_{3}-1},
\end{aligned}
$$

where $K_{n-1}$ is a function in the variables $p_{x}, p_{y}$ and $y p_{x}-Q x p_{y}$. Since $f_{n-1}$ must be a homogeneous polynomial of degree $n-1$ and $f_{n} \neq 0$ we have that

$$
\alpha_{1}=\alpha_{2}=\alpha_{3}=\alpha_{4}=0
$$

and

(15) $f_{n-1}=\left(x^{2}+y^{2}\right)^{m} \sum_{j_{4}+j_{5}+2 j_{6}=n-2 m-1} b_{j_{4}, j_{5}, j_{6}} p_{x}^{j_{4}} p_{y}^{j_{5}}\left(y p_{x}-Q x p_{y}\right)^{j_{6}}, \quad b_{j_{4}, j_{5}, j_{6}} \in \mathbb{C}$.

Now computing the terms of degree $n$ we obtain

$$
\begin{aligned}
& -\left(x^{2}+y^{2}\right)\left(p_{x} \frac{\partial f_{n-2}}{\partial x}+Q p_{y} \frac{\partial f_{n-2}}{\partial y}\right)+-2 m\left(x p_{x}+Q y p_{y}\right) f_{n-2}= \\
& \alpha_{0} f_{n}+p_{x} \frac{\partial f_{n}}{\partial x}+Q p_{y} \frac{\partial f_{n}}{\partial y}-x \frac{\partial f_{n}}{\partial p_{x}}-y \frac{\partial f_{n}}{\partial p_{y}}= \\
& \alpha_{0}\left(x^{2}+y^{2}\right)^{m} \sum_{j_{1}+j_{2}+2 j_{3}=n-2 m} a_{j_{1}, j_{2}, j_{3}} p_{x}^{j_{1}} p_{y}^{j_{2}}\left(y p_{x}-Q x p_{y}\right)^{j_{3}} \\
& \quad+\sum_{j_{1}+j_{2}+2 j_{3}=n-2 m} a_{j_{1}, j_{2}, j_{3}} p_{x}^{j_{1}-1} p_{y}^{j_{2}-1}\left(p_{x} y-p_{y} Q x\right)^{j_{3}-1}\left(x^{2}+y^{2}\right)^{m-1} \\
& \left(2 m p_{x} p_{y}\left(p_{x} y-p_{y} Q x\right)\left(p_{x} x+p_{y} Q y\right)+\left(x^{2}+y^{2}\right)\left(j_{1} p_{y} x\left(p_{y} Q x-p_{x} y\right)\right.\right. \\
& \left.\left.+p_{x} y\left(j_{3} p_{y}(Q-1) x+j_{2}\left(p_{y} Q x-p_{x} y\right)\right)\right)\right) .
\end{aligned}
$$


Solving this differential equation we obtain

(17)

$$
\begin{aligned}
f_{n-2}= & K_{n-2}\left(p_{x}, p_{y}, y p_{x}-Q x p_{y}\right)\left(x^{2}+y^{2}\right)^{m} \\
& +\left(x^{2}+y^{2}\right)^{m} \sum_{j_{1}+j_{2}+2 j_{3}=n-2 m} a_{j_{1}, j_{2}, j_{3}} p_{x}^{j_{1}} p_{y}^{j_{2}-1}\left(p_{x} y-p_{y} Q x\right)^{j_{3}-1} \\
& \left(p_{y}\left(\frac{m\left(p_{x} y-p_{y} Q x\right)}{x^{2}+y^{2}}-\frac{j_{3} p_{y}(Q-1) Q x}{p_{x}^{2}+p_{y}^{2} Q^{2}}\right)+\frac{2}{2 p_{x}\left(p_{x}^{2}+p_{y}^{2} Q^{2}\right)^{2}}\right. \\
& \left(\alpha_{0} p_{x} p_{y}\left(p_{x}^{2}+p_{y}^{2} Q^{2}\right)^{2}-\left(j_{2} p_{x}^{4}+\left(j_{2}+2 j_{3}\right) p_{y}^{2} Q^{2} p_{x}^{2}-\left(j_{1}+2 j_{3}\right) p_{y}^{2} Q p_{x}^{2}\right.\right. \\
& \left.\left.-j_{1} p_{y}^{4} Q^{3}\right)\left(p_{x} y-p_{y} Q x\right)\right) \arctan \left(\frac{p_{x} x+p_{y} Q y}{p_{y} Q x-p_{x} y}\right)+p_{x} p_{y}\left(\left(j_{2}+j_{3}\right) p_{y}^{2} Q^{3}\right. \\
& \left.+\left(j_{1}-j_{3}\right) p_{y}^{2} Q^{2}+\left(j_{2}-j_{3}\right) p_{x}^{2} Q+\left(j_{1}+j_{3}\right) p_{x}^{2}\right)\left(p_{x} y-p_{y} Q x\right) \\
& \left.\log \left(p_{x}^{2}\left(x^{2}+y^{2}\right)\right)\right)
\end{aligned}
$$

where $K_{n-2}$ is a function in the variables $p_{x}, p_{y}$ and $y p_{x}-Q x p_{y}$. Since $f_{n-2}$ must be a homogeneous polynomial of degree $n-2$ and $f_{n} \neq 0$ we get

$$
\alpha_{0}=j_{1}=j_{2}=j_{3}=0 .
$$

Then $n=2 m$ and since $f_{n-1}$ has degree $n-1$ it follows from (14), (15) and (17) that $f_{n}=a_{0,0,0}\left(x^{2}+y^{2}\right)^{m}, f_{n-1}=0, f_{n-2}=m a_{0,0,0}\left(x^{2}+y^{2}\right)^{m-1}=\left(\begin{array}{c}m \\ 1\end{array}\right) a_{0,0,0}\left(x^{2}+y^{2}\right)^{m-1}$.

Computing the terms of degree $n-1$ we get

$$
-\left(x^{2}+y^{2}\right)\left(p_{x} \frac{\partial f_{n-3}}{\partial x}+Q p_{y} \frac{\partial f_{n-3}}{\partial y}\right)=-2 m\left(x p_{x}+Q y p_{y}\right) f_{n-3} .
$$

Solving this differential equation we obtain

$$
f_{n-3}=K_{n-3}\left(p_{x}, p_{y}, y p_{x}-Q x p_{y}\right)\left(x^{2}+y^{2}\right)^{m},
$$

where $K_{n-3}$ is a function in the variables $p_{x}, p_{y}$ and $y p_{x}-Q x p_{y}$. Since $f_{n-3}$ must be a homogeneous polynomial of degree $n-3$ and $n=2 m$, we get that $f_{n-3}$ must be a homogeneous polynomial of degree $2 m-3$. This is not possible and then $K_{n-3}=0$ which yields $f_{n-3}=0$.

Computing the terms of degree $n-2$ we get

$$
\begin{gathered}
-\left(x^{2}+y^{2}\right)\left(p_{x} \frac{\partial f_{n-4}}{\partial x}+Q p_{y} \frac{\partial f_{n-4}}{\partial y}\right)+2 m\left(x p_{x}+Q y p_{y}\right) f_{n-4} . \\
=p_{x} \frac{\partial f_{n-2}}{\partial x}+Q p_{y} \frac{\partial f_{n-2}}{\partial y}-x \frac{\partial f_{n-2}}{\partial p_{x}}-y \frac{\partial f_{n-2}}{\partial p_{y}} \\
=2 a_{0,0,0}\left(\begin{array}{c}
m \\
1
\end{array}\right)(m-1)\left(x^{2}+y^{2}\right)^{m-2}\left(x p_{x}+y Q p_{y}\right) .
\end{gathered}
$$


Solving it we obtain

$$
f_{n-4}=K_{n-4}\left(p_{x}, p_{y}, y p_{x}-Q x p_{y}\right)\left(x^{2}+y^{2}\right)^{m}+\frac{m(m-1)}{2} a_{0,0,0}\left(x^{2}+y^{2}\right)^{m-2},
$$

where $K_{n-4}$ is a function in the variables $p_{x}, p_{y}$ and $y p_{x}-Q x p_{y}$. Since $f_{n-4}$ must be a homogeneous polynomial of degree $n-4=2 m-4$ we must have $K_{n-4}=0$ and $f_{n-4}=\left(\begin{array}{c}m \\ 2\end{array}\right) a_{0,0,0}\left(x^{2}+y^{2}\right)^{m-2}$.

Proceeding inductively we get that

$$
f_{n-2 k-1}=0 \quad \text { for } k=0, \ldots, m-1
$$

and

$$
f_{n-2 k}=\left(\begin{array}{c}
m \\
k
\end{array}\right) a_{0,0,0}\left(x^{2}+y^{2}\right)^{m-k} \quad \text { for } k=0, \ldots, m .
$$

This implies that $f_{n}=a_{0,0,0}\left(1+x^{2}+y^{2}\right)^{m}$. Thus the unique irreducible Darboux polynomial of equation (2) is $1+x^{2}+y^{2}$. This concludes the proof of the theorem.

\section{Proof of Theorem 3}

To prove Theorem 3 we recall two auxiliary results. The first one was proved in [6] while the second one was proved in [8].

Lemma 8. Let $f$ be a polynomial and $f=\prod_{j=1}^{s} f_{j}^{\alpha_{j}}$ its decomposition into irreducible factors in $\mathbb{C}[x, y, z]$. Then $f$ is a Darboux polynomial if and only if all the $f_{j}$ are Darboux polynomials. Moreover, if $K$ and $K_{j}$ are the cofactors of $f$ and $f_{j}$, then $K=\sum_{j=1}^{s} \alpha_{j} K_{j}$

Lemma 9. The existence of a rational first integral for a polynomial differential system (2) implies the existence of a polynomial first integral, or the existence of two Darboux polynomials with the same non-zero cofactor.

The proof of Theorem 3 follows readily from Theorems 2 and 4 together with Lemmas 8 and 9 .

\section{Exponential Factors: Proof of Theorem 5}

To prove Theorem 5 we will use the following known result whose proof and geometrical meaning is given in $[2,10]$.

Proposition 10. The following statements hold.

(a) If $E=\exp \left(g_{0} / g_{1}\right)$ is an exponential factor for the polynomial system (2) and $g_{1}$ is not a constant polynomial, then $g_{1}=0$ is an invariant algebraic hypersurface.

(b) Eventually $e^{g_{0}}$ can be exponential factors, coming from the multiplicity of the infinite invariant hyperplane. 
The following result given in $[2,10]$ characterizes the algebraic multiplicity of an invariant algebraic hypersurface using the number of exponential factors of system (2) associated with the invariant algebraic hypersurface.

Theorem 11. Given an irreducible invariant algebraic hypersurface $g=0$ of degree $m$ of system (2), it has algebraic multiplicity $k$ if and only if the vector field associated to system (2) has $k-1$ exponential factors of the form $\exp \left(g_{i} / g^{i}\right)$, where $g_{i}$ is a polynomial of degree at most im and $\left(g_{i}, g\right)=1$ for $i=1, \ldots, k-1$.

In view of Theorem 11 if we prove that $e^{g_{0} / g}$ is not an exponential factor with degree $g_{0} \leq$ degree $g$, there are no exponential factors associated to the invariant algebraic hypersurface $g=0$.

System (2) has the irreducible Darboux polynomial $1+x^{2}+y^{2}$. Then in view of Proposition 10 it can have an exponential factor of the form: either $E=\exp (g)$ with $g \in \mathbb{C}\left[x, y, p_{x}, p_{y}\right] \backslash \mathbb{C}$, or $E=\exp \left(g /\left(1+x^{2}+y^{2}\right)^{m}\right)$ with $m \geq 1$ and such that $g \in \mathbb{C}\left[x, y, p_{x}, p_{y}\right]$ and is coprime with $1+x^{2}+y^{2}$. We first prove that system (2) has no exponential factors of the form $E=\exp \left(g /\left(1+x^{2}+y^{2}\right)^{m}\right)$.

Assume that system (2) has an exponential factor of the form $E=\exp \left(g /\left(1+x^{2}+\right.\right.$ $\left.\left.y^{2}\right)^{m}\right)$ with $m \geq 1$ such that $1+x^{2}+y^{2}$ is coprime with $g \in \mathbb{C}\left[x, y, p_{x}, p_{y}\right]$. In view of Theorem 11 we can assume that $m=1$ and that $g$ has degree at most two (note that here $g=1+x^{2}+y^{2}$ has degree two). We write $g$ as a polynomial of degree two in the variables $x, y, p_{x}, p_{y}$ as follows

$$
\begin{aligned}
g= & a_{0}+a_{1} x+a_{2} y+a_{3} p_{x}+a_{4} p_{y}+a_{5} x^{2}+a_{6} x y+a_{7} x p_{x}+a_{8} x p_{y} \\
& +a_{9} y^{2}+a_{10} y p_{x}+a_{11} y p_{y}+a_{12} p_{x}^{2}+a_{13} p_{x} p_{y}+a_{14} p_{y}^{2} .
\end{aligned}
$$

Clearly, $g$ satisfies

$$
\begin{aligned}
& -\left(1+x^{2}+y^{2}\right) p_{x} \frac{\partial g}{\partial x}-Q p_{y}\left(1+x^{2}+y^{2}\right) \frac{\partial g}{\partial y}+x \frac{\partial g}{\partial p_{x}}+y \frac{\partial g}{\partial p_{y}} \\
& +2\left(x p_{x}+Q y p_{y}\right) g=L\left(1+x^{2}+y^{2}\right)
\end{aligned}
$$

where $L$ is a polynomial of degree two in the variables $x, y, p_{x}, p_{y}$. Setting

$$
\begin{aligned}
L= & b_{0}+b_{1} x+b_{2} y+b_{3} p_{x}+b_{4} p_{y}+b_{5} x^{2}+b_{6} x y+b_{7} x p_{x}+b_{8} x p_{y} \\
& +b_{9} y^{2}+b_{10} y p_{x}+b_{11} y p_{y}+b_{12} p_{x}^{2}+b_{13} p_{x} p_{y}+b_{14} p_{y}^{2}
\end{aligned}
$$

in (19) with an algebraic manipulator we conclude that

$$
g=a_{9}\left(1+x^{2}+y^{2}\right) \text { and } L=0 .
$$

However this is not possible since $g$ is coprime with $1+x^{2}+y^{2}$.

In summary, if (2) has an exponential factor it must be of the form $E=\exp (g)$ with $g \in \mathbb{C}\left[x, y, p_{x}, p_{y}\right] \backslash \mathbb{C}$. In this case, $g$ satisfies

$$
-\left(1+x^{2}+y^{2}\right) p_{x} \frac{\partial g}{\partial x}-Q p_{y}\left(1+x^{2}+y^{2}\right) \frac{\partial g}{\partial y}+x \frac{\partial g}{\partial p_{x}}+y \frac{\partial g}{\partial p_{y}}=L,
$$

where $L=L\left(x, y, p_{x}, p_{y}\right)$ is some polynomial of degree two in the variables $x, y, p_{x}, p_{y}$ and that we can take as in (20). 
We write $g$ as $g=\sum_{j=0}^{n} g_{j}\left(x, y, p_{x}, p_{y}\right)$ where each $g_{j}$ is a homogeneous polynomial of degree $j$. Without loss of generality we can assume that $g_{n} \neq 0$ with $n>0$.

Assume $n \geq 3$. Then computing the terms of degree $n+2$ in (21) we get (8). Now proceeding as we did in the proof of Theorem 2 we get $g_{n}$ is as in (9). Then the terms of degree $n$ in (21) (since $n \geq 3$ ) they satisfy equation (10) which, again in view of the proof of Theorem 2 they must be zero. Then $g$ has degree at most two. In this case we write it as in (18). Then imposing that $g$ satisfies (21) and solving it with an algebraic manipulator we conclude that

$$
g=a_{0}+b_{1} p_{x}+\frac{b_{7}}{2} p_{x}^{2}+a_{4} p_{y}+b_{8} p_{x} p_{y}+a_{14} p_{y}^{2}+a_{10}\left(y p_{x}-Q x p_{y}\right) .
$$

This concludes the proof of the theorem.

\section{Proof of Theorem 6}

In order to proof Theorem 6 we need the following result whose proof is given in $[6]$.

Theorem 12. Suppose that system (2) admits p Darboux polynomials and with cofactors $K_{i}$ and $q$ exponential factors $F_{j}$ with cofactors $L_{j}$. Then there exists $\lambda_{j}, \mu_{j} \in \mathbb{C}$ not all zero such that

$$
\sum_{i=1}^{q} \lambda_{k} K_{i}+\sum_{i=1}^{q} \mu_{i} L_{i}=0
$$

if and only if the function $G$ given in (7) (called of Darboux type) is a first integral of system (2).

In view of Theorem 12 to characterize the Darboux first integrals we need to compute the Darboux polynomials and the exponential factors. Then, using Theorems 2, 4 and 5 if $G$ is a Darboux first integral of system (2) it must be of the form (7), i.e.

$$
G=\left(1+x^{2}+y^{2}\right)^{\lambda} e^{\mu_{1} p_{x}+\mu_{2} p_{x}^{2}+\mu_{3} p_{x} p_{y}+\mu_{4} p_{y}+\mu_{5} p_{y}^{2}+\mu_{6}\left(y p_{x}-Q x p_{y}\right)}
$$

and the cofactors must satisfy

$-2 \lambda\left(x p_{x}+Q y p_{y}\right)+\mu_{1} x+2 \mu_{2} x p_{x}+\mu_{3}\left(x p_{y}+y p_{x}\right)+\mu_{4} y+2 \mu_{5} y p_{y}+\mu_{6}(1-Q) x y=0$.

Solving this system we have either $\mu_{1}=\mu_{3}=\mu_{4}=\mu_{6}=0$ and $\mu_{2}=\lambda, \mu_{5}=Q \lambda$. From (3) this yields

$$
G=\left[\left(1+x^{2}+y^{2}\right) e^{\left(p_{x}^{2}+Q p_{y}^{2}\right)}\right]^{\lambda}=H_{0}^{\lambda}
$$

That is, all the Darboux first integrals of system (2) are Darboux functions in the variable $H_{0}$. This concludes the proof of Theorem 6 . 


\section{ACKNowledgements}

The first author is supported by the grants MINECO/FEDER MTM 2008-03437, Generalitat de Catalunya 2009SGR410, ICREA Academia and FP7-PEOPLE-2012IRSES-316338 and 318999. The second author was supported by AGAUR grant PIVDGR-2010 and by FCT through the project PTDC/MAT/117106/2010 and through CAMGSD, Lisbon.

\section{REFERENCES}

[1] R. Abraham and J.E. Marsden, Foundations of Mechanics, Benjamin, Reading, Massachussets, 1978.

[2] C. Christopher, J. Llibre and J.V. Pereira, Multiplicity of invariant algebraic curves in polynomial vector fields, Pacific J. Math. 229 (2007), 63-117.

[3] G. Contopoulos And J. Seimenis, Application of the Prendergast method to a logarithmic potential, Astron. Astrophys. 227 (1990), 49-53.

[4] G. Darboux, Mémoire sur les équations différentielles du premier ordre et du premier degreé (Mélanges), Bull. Sci. Math. 2éme série 2 (1878), 60-96, 123-144, 151-200.

[5] G. Darboux , De l'emploi des solutions particulières algébriques dans l'intégration des systèmes d'équations différentielles algébriques, C. R. Math. Acad. Sci. Paris 86 (1878), 10121014.

[6] F. Dumortier, J. Llibre And J.C. Artés, Qualitative theory of planar differential systems, Universitext, Springer-Verlag, Berlin, 2006.

[7] L. Jimenez-Lara And J. Llibre, The cored and logarithmic galactic potentials: Periodic orbits and integrability, J. Math. Phys. 53, 042901 (2012); doi: 10.1063/1.3697838.

[8] J. Llibre And C. Valls, On the integrability of the Bianchi IX system, J. Math. Phys. 46 (2005), 072901-13 pp.

[9] J. Llibre AND X. ZHANG, Darboux theory of integrability in $\mathbb{C}^{n}$ taking into account the multiplicity, J. Differential Equations 246 (2009), 541-551.

[10] J. LlibRe AND X. Zhang, Darboux theory of integrability for polynomial vector fields in $\mathbb{R}^{n}$ taking into acocunt their multiplicity at infinity, Bull. Sci. math 133 (2009), 765-778.

[11] Y. PAPAPhILIPPOU AND J. LASKAR, Frequency map analysis and global dynamics in a two degrees of freedom galactic potential, Astron. Astrophys. 307 (1996), 426-449.

[12] G. Pucacco, D. Boccaletti And C. Belmonte, Quantitative predictions with detuned normal forms, Celest. Mech. Dyn. Astron. 102 (2008), 163-176.

[13] G. Pucacco, D. Boccaletti And C. Belmonte, Periodic orbits in the logarithmic potential, Astron. Astrophys. 489 (2008), 1055-1063.

${ }^{1}$ Departament de Matemàtiques, Universitat Autònoma de Barcelona, 08193 Bellaterra, BArcelona, Catalonia, Spain

E-mail address: jllibre@mat.uab.cat

2 Departamento de Matemática, Instituto Superior Técnico, Universidade Técnica De Lisboa, Av. Rovisco Pais 1049-001, Lisboa, Portugal

E-mail address: cvalls@math.ist.utl.pt 